\title{
New Quantitative Phase Reconstruction Technique using Hollow-cone Probe and Annularly Arrayed Detectors in STEM
}

\author{
Takafumi Ishida ${ }^{1}$, Tadahiro Kawasaki ${ }^{2,3,4}$, Takayoshi Tanji ${ }^{2,4}$ and Takashi Ikuta ${ }^{5}$ \\ 1. Department of Electrical Engineering and Computer Science, Nagoya University, Nagoya 464-8603 \\ Japan \\ 2. EcoTopia Science Institute, Nagoya University, Nagoya 464-8603 Japan \\ 3. Nanostructures Research Laboratory, Japan Fine Ceramics Center, Nagoya 456-8587 Japan \\ 4. Global Research Center for Environment and Energy based on Nanomaterials Science, Nagoya, 464- \\ 8603 Japan \\ 5. Department of Electrical and Electronic Engineering, Osaka Electro-Communication University, \\ Neyagawa 572-8530 Japan
}

The quantitative evaluation of the specimen structure in an image requires a projected potential image. The established method for evaluating the electrostatic potential in a specimen is electron holography which can directly reconstruct the phase of electron waves. In scanning transmission electron microscopy (STEM), a phase reconstruction technique that employs a multi-channel detector has been proposed [1,2]. The resolution of a reconstructed phase image based on the use of a multi-channel detector can be twice that of a conventional bright-field image. This means that we may expect higher resolution than electron holography. The annular bright-field phase (ABFP) imaging technique proposed by the present authors [3], which is a phase reconstruction technique using a multi-channel detector, has been demonstrated to be capable of high-resolution phase imaging. The reconstructed phase image obtained by ABFP imaging enables that quantification of the projected potential [4].

Figure 1 shows a schematic of ABFP imaging. A hollow-cone probe is formed with an annular objective aperture, and multiple images are simultaneously recorded with annularly arrayed detectors (AADs). Each image contains different information, because the AADs capture waves diffracted at different azimuthal angles. The phase image is reconstructed by the following processes. The images obtained by AADs are Fourier transformed. Then, a ring-shaped band pass filter is applied to each Fourier spectrum to extract the linear imaging components. Finally, the sum of these filtered spectra is inversely Fourier transformed to obtain the real and imaginary parts of the electron wave. The reconstructed phase image is determined by the arctangent of the ratio of the imaginary and real parts of the reconstructed complex amplitude wave.

In order to evaluate quantitatively ABFP imaging, we performed the multislice image simulation. Figure 2(a) is an example of the simulated phase image of a single atom $(\mathrm{Cu})$ reconstructed using ABFP imaging. Images were calculated for single neutral atoms of atomic numbers from 1 to 103 . Since the absolute value of the phase shift cannot be determined in our reconstruction method, the relative phase shift measured by the height from the negative bottom to the positive top of the peak was defined as the maximum phase shift, as shown in Fig. 2(b). Figure 2(c) shows the maximum phase shifts for single atoms as a function of atomic number $Z$ in ABFP imaging. A least-squares fitting curve shows that the maximum phase shifts are proportional to approximately $Z^{0.6}$. The result of Fig. $2(\mathrm{c})$ is in good agreement with the simulated results of phase shifts obtained by electron holography [5]. This indicates that the projected potential was determined using the maximum phase shift, though it is a relative value. The reconstruction method can also be applied to thin crystal samples, and has the capability of counting the number of atoms in columns. 
In conclusion, we have developed a new phase reconstruction technique for high-resolution in STEM. This technique was quantitatively evaluated using image simulations. The results showed that the phase shifts caused by single atoms were proportional to $\mathrm{Z}^{0.6}$. Comparisons of experimental and simulated results for thin crystal samples will be discussed [6].

References:

[1] M Landauer et al., Optik 100 (1995), p. 37

[2] M. Taya et al., Rev. Sci. Instrum. 78 (2007), p. 083705

[3] T. Ishida et al., Microscopy (in press) doi: $10.1093 / \mathrm{jmicro} / \mathrm{dfu} 098$

[4] T. Ishida et al., Microscopy (in press) doi: 10.1093/jmicro/dfu113

[5] H. Lichite et al., Annu. Rev. Mater. Res. 37 (2007) p. 539

[6] This work was supported by JSPS KAKENHI Grant Numbers 18GS0211 and 24360020, and in part by the program "Global Research Center for Environment and Energy based on Nanomaterials Science" of MEXT, Japan.

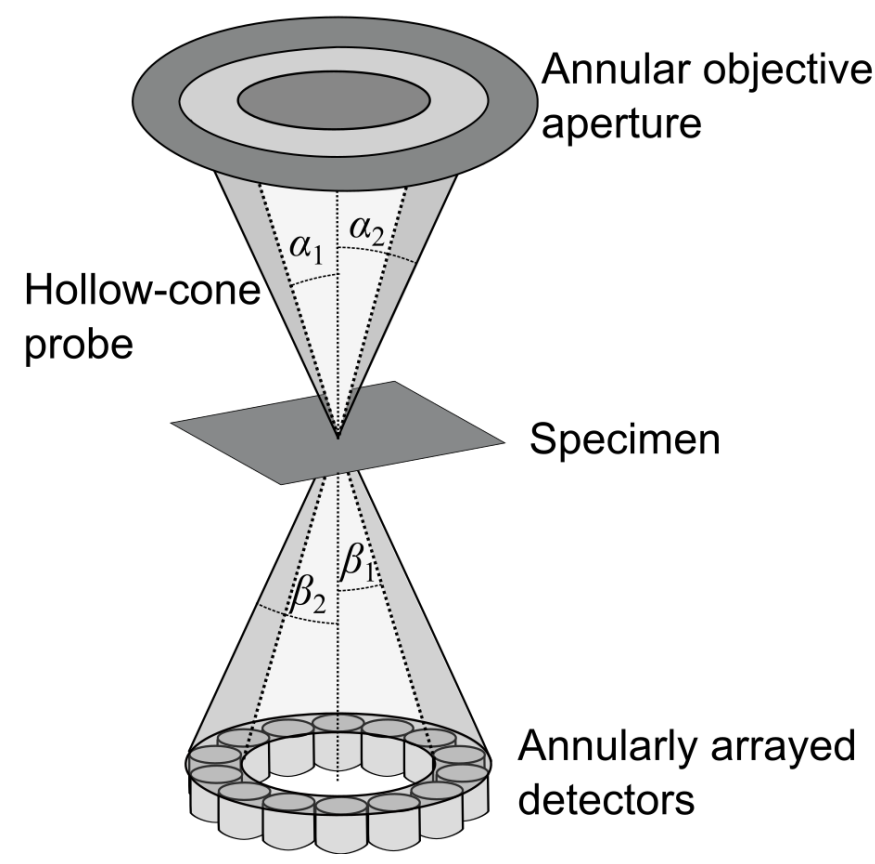

Figure 1. Schematic diagram of an STEM instrument for ABFP imaging. The hollow-cone probe is formed by an objective lens using an annular aperture, and the different azimuthal angle electrons that pass through the specimen are simultaneously acquired by the annularly arrayed detectors. The annular objective aperture inner and outer semi-angles $\alpha_{1}$ and $\alpha_{2}$ are determined so as to be coincident with the inner and outer angles $\beta_{1}$ and $\beta_{2}$ of the annularly arrayed detectors in the detection plane.

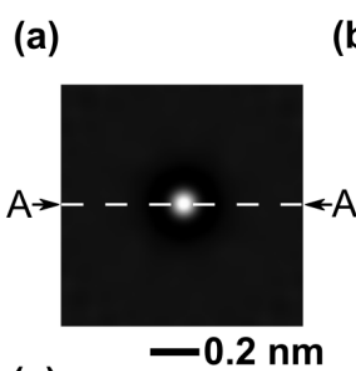

(b)

(c)
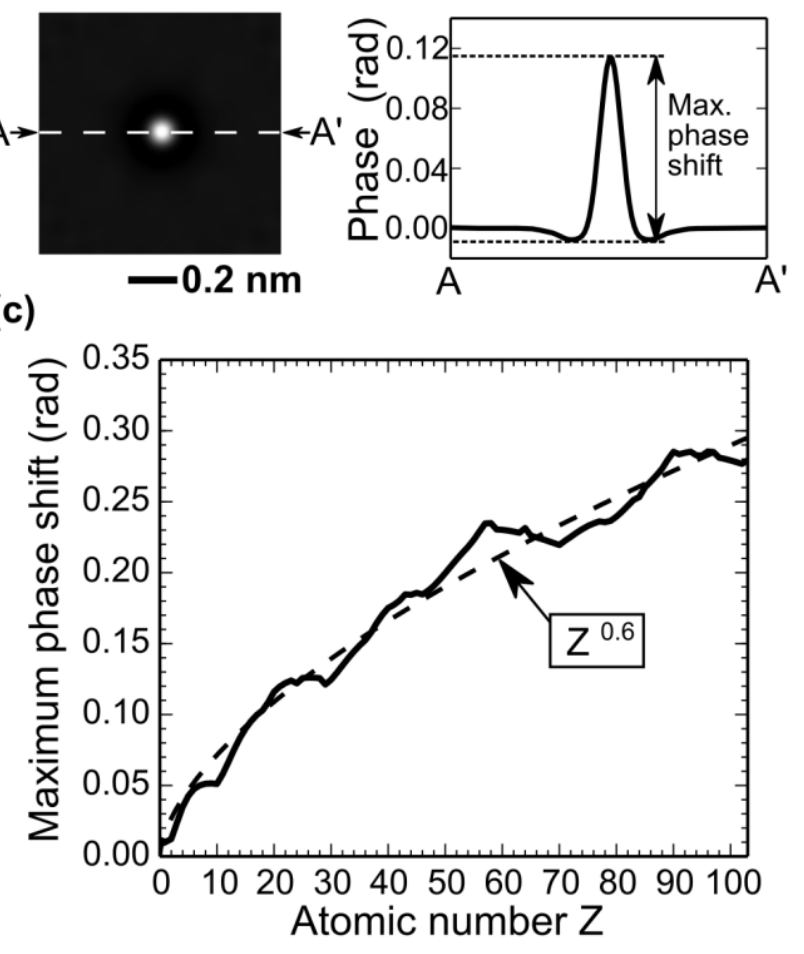

Figure 2. (a) ABFP image of a single atom (copper). An estimated probe size is $0.1 \mathrm{~nm}\left(V_{a}\right.$ $=200 \mathrm{kV}$ ). (b) Line profile of (a) (A-A'). (c) Maximum phase shift due to a single atom as a function of atomic number. The dashed line is a least-squares fitting curve. The simulated phase shift is proportional to $Z^{0.6}$. 